\title{
Preliminary Investigation of Accelerating Molecular Dynamics Simulation on Godson-T Many-Core Processor
}

\author{
Liu Peng ${ }^{1}$, Guangming Tan ${ }^{2}$, Rajiv K. Kalia ${ }^{1}$, Aiichiro Nakano ${ }^{1}$, \\ Priya Vashishta ${ }^{1}$, Dongrui Fan ${ }^{2}$, and Ninghui Sun ${ }^{2}$ \\ ${ }^{1}$ Collaboratory for Advanced Computing and Simulation, \\ University of Southern California, Los Angeles, CA, USA \\ \{liupeng, rkalia, anakano, priyav\}@usc.edu \\ 2 Institute of Computing Technology, \\ Chinese Academy of Sciences, Beijing, China \\ \{tgm, fandr, snh\}@ict.ac.cn
}

\begin{abstract}
Molecular dynamics (MD) simulation is widely used in computational science, however, its irregular memory-access pattern imposes great difficulty on performance optimization. This paper presents a joint application/architecture study to accelerate MD on an emerging unconventional computing platformGodson-T many-core architecture. We propose three incremental optimizations: (1) a divide-and-conquer algorithm adaptive to on-chip memory; (2) a novel datalayout to re-organize linked-list cell data structures to improve data locality; (3) an on-chip locality-aware parallel algorithm to enhance data reuse. Experiments on an event-driven, cycle-accurate Godson-T simulator achieve excellent speedup of 62 on 64 cores.
\end{abstract}

\section{Introduction}

Molecular dynamics (MD) simulation is widely used to study material properties at the atomistic level. But increasingly large computing power is needed to satisfy the spatiotemporal scale of the real world simulations. The advent of many-core paradigm has provided unprecedented computing power, and promises to enable large-scale and longtime simulation only if we can efficiently harvest the computing power. Challenges to achieve efficient parallel MD algorithm mainly on many-core platform arise from two aspects: (1) MD application is characterized by irregular memory access which imposes difficulty on locality optimization; (2) many-core hardware limitation (volume of onchip memory, bandwidth of on-chip networking, etc.) constrains the size of working-set per core which imposes difficulty on on-chip parallelization. To address these difficulties, this paper presents a joint study from both application and architecture aspects on how to accelerating MD on Godson-T emerging many-core architecture, where we map an MD algorithm to architecture for achieving high on-chip parallel efficiency.

The main contribution of this paper are: (1) An adaptive divide-and-conquer (ADC) algorithm is designed to optimize the use of memory hierarchy; (2) A novel data layout is employed to re-organize linked-list cell data structures to maximize data locality; 
(3) An on-chip locality-aware parallel algorithm is designed to maximize data reuse;

(4) Detailed experiments on an Godson-T simulator shows the optimized MD achieves a strong-scaling parallel efficiency 0.92 on 64 cores.

The rest of this paper is organized as follows. Section 2 briefly introduces the DCMD algorithm used in this work, where key performance bottlenecks are summarized. Section 3 highlights the main architectural features of Godson-T, and Section 4 describes our optimization strategies. Section 5 presents the experimental results and detailed analysis. Finally, Section 6 concludes the paper.

\section{A Divide-and-Conquer MD Algorithm}

We have previously proposed a space-time multiresolution MD (MRMD) algorithm to reduce the $O\left(N^{2}\right)$ time complexity of potential evaluation to $O(N)$ [4]. In the MRMD, $E\left(r^{N}\right)$ consists of two-body $E_{2}\left\{r_{i j}\right\}$ and three-body $E_{3}\left\{r_{i j k}\right\}$ terms within a cutoff radius $r_{c}$. In the linked-list cell method, the dimension $R_{c}$ of the cells is usually chosen to be larger than $r_{c}$. For a given atom in a cell, the search space for interacting neighbor atoms is limited to the 26 nearest neighbor cells. Conventional summation rule to compute 3-body interaction is written as $E_{3}\left(r_{i j k}\right)=\sum_{i=1}^{N} \sum_{j=1}^{n b r(i)} \sum_{k \neq i}^{n b r(j)} v\left(r_{i}, r_{j}, r_{k}\right)$, where $r_{i}$ is the coordinate of the $i-t h$ atom and $n b r(i)$ is the list of neighbor atoms within the three-body cutoff length from atom $i$, which acts as the center of atomic triplet $(j, i, k)$. To maximally exploit parallelism in a multi-core cluster, our EDCSTEP-HCD scheme has employed a multi-level parallelization strategy [5]6]. Although this scheme has achieved internode parallel efficiency well over 0.95 for 218 billionatom MD simulation on 212, 992 BlueGene/L processors [5], it suffers inefficient onchip parallelism with on-chip parallel efficiency only 0.65 for 8 threads on a dual Intel quadcore Xeon SMP platform [6]. We observe several features that prevent the program from achieving high performance on conventional multi-core architectures: (1)Irregular memory access. Straightforward or sparse-matrix-like implementation of linked-list data structure leads to irregular memory accesses in three-body interaction calculation;

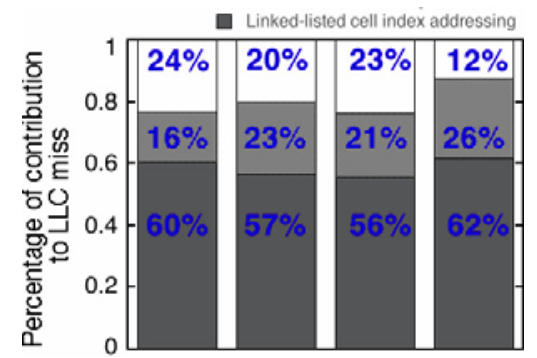

(a)

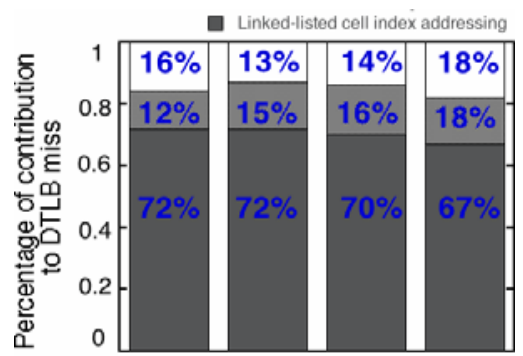

(b)

Fig. 1. (a) and (b) are percentages of events that cause last level cache (LLC) miss and data translation look aside buffer (DTLB) miss for the original DC-MD algorithm on an Intel quadcore core i7 920 platform measured by Intel VTune Performance Analyzer 
(2)High latency to access shared data. Let $N_{c}$ denote the number of cells in each Cartesian direction, $q$ the atom density (number of atoms divided by system volume $\left(N_{c} R_{c}\right)^{3}$, then the total number of cross-cell pairs in the system is given by $N_{c}^{3} \times q R_{c}^{3} \times 26 q R_{c}^{3}$. Thereby the size of atomic data for interaction computation easily exceeds that of the last level cache. Combining with the irregular memory access mentioned before, the latency problem becomes even worse, as evidenced by the memory accessing performance in Fig. 1(a) and Fig. 1(b) conducting on Intel quadcore core i7 920 platform measured by the Intel VTune Performance Analyzer. Therefore, it is of great significance to optimize the memory accessing to improve the performance and scalability of our MD application on many-core platforms.

\section{Godson-T Many-Core Architecture}

Godson-T is a low-power many-core architecture developed by Institute of Computing Technology, Chinese Academy of Sciences to serve as a dedicated petaflops computing engine. As shown in Fig. 2, Godson-T has 64 homogeneous, dual-issue and in-order processing cores running at $1 \mathrm{GHz}$, where a floating-point multiply-accumulate operation can be issued to a fully-pipelined function unit in each cycle, resulting in a peak floating-point performance of 128 Gflops. The 8-pipeline processing core supports 32bit MIPS ISA (64-bit ISA will be supported in latter version) with synchronization instruction extensions. Key architectural features for achieving decent scalability and high performance include the following [1]:

- Fine-grained parallelism [8]. Each core works as a lightweight hardware thread unit executing in a non-preemptive manner. A dedicated synchronization manager (SM) is a centralized unit to collect and handle synchronization requests, which provides architectural support for fast mutual exclusion, barrier and signal/wait synchronization. In addition, an extremely efficient thread execution runtime system has been developed to manage thread execution [8]3].

- Locality-awareness. Each processing core has a 16KB 2-way set-associative private instruction cache and a $64 \mathrm{~KB}$ local memory (like data cache). As inspired by

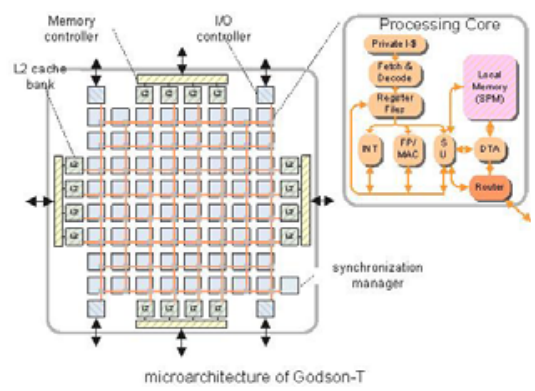

Fig. 2. Godson-T architecture: INT is fixed point arithmetic unit, FP/MAC is floating point unit, and $\mathrm{CU}$ is communication unit

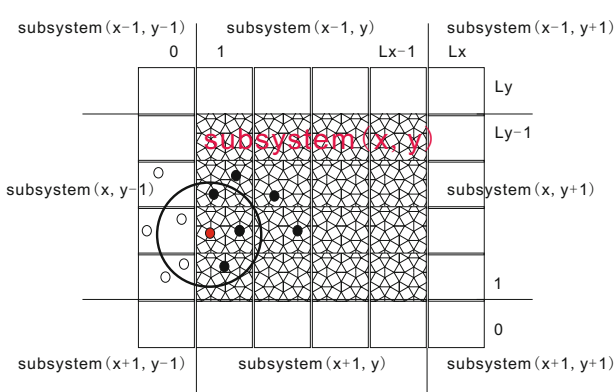

Fig. 3. Cellular decomposition scheme for on-chip parallelization. Cached atoms and resident atoms are shown in white and shaded boxes 
IBM CELL and Nvidia GPU, an explicit memory hierarchy is implemented for user to exploit better locality with less complex hardware implementation compared to that of the traditional transparent memory hierarchy. Moreover, in Godson-T, each local memory is configured as explicitly-controlled, globally-addressed scratchedpad memory (SPM) to further help programmer maximize locality. An $8 \times 8$ packetswitching 2D mesh network connects all on-chip units with a 128bit bandwidth employing deterministic X-Y routing policy, which can provide a total of $2 \mathrm{~TB} / \mathrm{s}$ on-chip bandwidth among 64 processing cores. In addition, there are 16 addressinterleaved L2 cache banks (256KB each) distributed along the perimeter of the chip, which are shared by all processing cores and can serve up to 64 cache accessing requests in total. The bandwidth between SPM and L2 cache is $256 \mathrm{~GB} / \mathrm{s}$, and each four L2 cache banks on the same side of the chip share a memory controller with a $25.6 \mathrm{~GB} / \mathrm{s}$ memory-accessing bandwidth.

- Latency tolerance [7]. Since there may exist intensive contention on on-chip network and memory controller, latency to L2 cache will possibly become primary obstacle to achieve decent performance. To address this issue, a DMA (direct memory accessing)-like coprocessor Data Transfer Agent (DTA) is built in each core to do fast data communication, that is, when one core is doing calculations, DTA can be programmed to manage various data communications at backend in parallel.

\section{Optimizations on Godson-T}

In this section, we describe how to design an efficient MD algorithm based on the features provided by Godson-T many-core architecture.

\subsection{Adaptive Divide-and-Conquer Algorithm}

In the original DC-MD algorithm, the physical space is subdivided into spatially localized cells, with local atoms constituting subproblems. The algorithm recursively divides a coarse cell into finer cells until some criterion is satisfied. In our adaptive divide-andconquer (ADC) algorithm specially designed for many-core architectures, we use the size of the first level memory (i.e. private local memory), $C_{p m}$, as a critical factor of the criterion.

Since this paper only addresses the issue of fine-grained parallelism within a subsystem, we assume that the interchange of cached atoms (atoms near subsystem boundaries) has been completed by a higher-level parallelism (e.g. using MPI) among multiple many-core computing processors [5]. Figure 3 illustrates of the cells in a subsystem. The algorithm divides the subsystem consisting of the resident and cached atoms into small cells of equal size. Assume that there are $P=P_{x} \times P_{y} \times P_{z}$ cores in a many-core processor and that the number of cells in a subsystem is $L=L_{x} \times L_{y} \times L_{z}$. Then each core $i$ processes $\frac{L}{P}$ cells as Eq. 1 (since how to efficiently embed 3D mesh into $2 \mathrm{D}$ one has already been solved by classical algorithms [2], the 2D on-chip network on Godson- $T$ is viable for this decomposition): $\left\{\left(c_{x}, c_{y}, c_{z}\right) \mid c_{x} \in\left[\frac{i L_{x}}{P_{x}}, \frac{(i+1) L_{x}}{P_{x}}\right), c_{y} \in\right.$ $\left.\left[\frac{i L_{y}}{P_{y}}, \frac{(i+1) L_{y}}{P_{y}}\right), c_{z} \in\left[\frac{i L_{z}}{P_{z}}, \frac{(i+1) L_{z}}{P_{z}}\right)\right\}$. Let $B$ denote the memory space for storing one 
atomic data, $N_{b}$ denote the number of neighbor atoms per cell. In order for all the resident atomic data to fit in the private local memory, $R_{c}$ should satisfy

$$
q R_{c}^{3} \times\left(N_{b}+\frac{L}{P}\right) \times B \leq C_{p m} \Rightarrow R_{c} \leq \sqrt[3]{\frac{P C_{p m}}{\left(P N_{b}+L\right) B q}} .
$$

ADC algorithm performs recursive cellular decomposition until Eq. 1 is satisfied. When the atomic data are distributed into each core's local memory, they are reused in both two- and three-body force calculations, as the software controlled SPM provides a mechanism for user to decide what data locate in the private local memory and when. However, a direct implementation based on the linked-list cell data structure is not efficient enough because of MD's irregularity. In the next subsection, we propose a novel data layout optimization to address this problem.

\subsection{Data Layout Optimization}

At the beginning of MD simulation, each atom is assigned an integer in [0... -1$]$, which is used as an identifier for the linked-list based algorithm to access atomic data. We refer to this method as global-ID-centered addressing. However, during the simulation, the identifiers cannot be kept contiguous due to atom migration between computing nodes/processors. In the ADC algorithm, it is expected that the atomic data is distributed among different cores, where each cell only interacts with 26 neighbor cells. Therefore, if all the atomic data within one cell were grouped together, they would be easily reached through its cell index. Here, we propose a new strategy-cell-centered addressing.

Figure 4(a) depicts the data structure designed for the atomic data in SPM, where na denotes the maximum number of atoms in one cell. Since all the atomic data for each cell are grouped, the cell index $(c c)$ can be used to search the neighbor cells, and then the

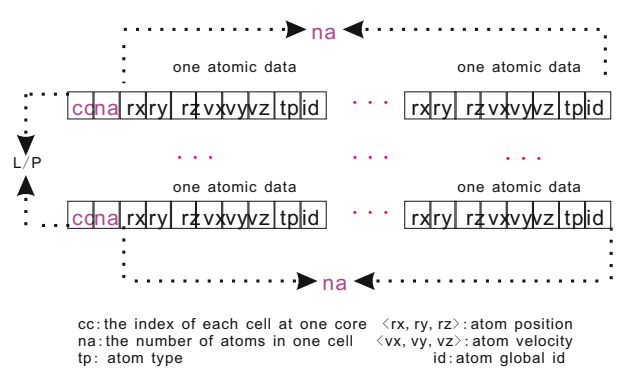

(a)

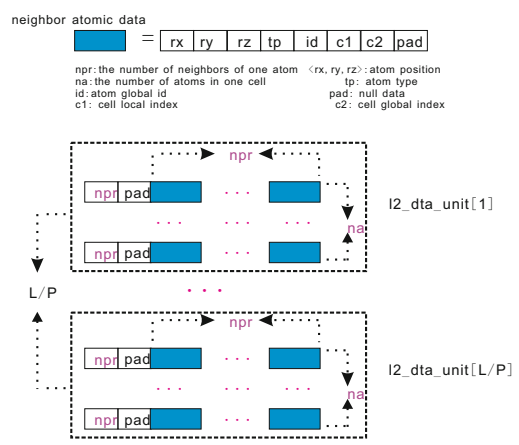

(b)

Fig. 4. (a) The atomic data of cells in a core's SPM. (b)The neighbor atomic data of cells in the shared L2 cache or off-chip memory. Pad is used for address alignment. Each L2_data_unit $i$ ] represents a contiguous block of all neighbor atomic data for the $i-t h$ cell in L2 cache or off-chip memory $(i=1, \ldots, L / P)$. 
atomic data in each cell can be touched contiguously. Moreover, considering the sequential mapping between cores and cells, the searching of neighbor cells is completed in $O(1)$ time: The scalar value of $c c$ is transformed into a vector $\left(c c_{x}, c c_{y}, c c_{z}\right)$, then the neighbor cell index is calculated by the combination of $\left\{\left(c c_{x}+l_{x}, c c_{y},+l_{y}, c c_{z}+l_{z}\right) \mid l_{x}, l_{y}, l_{z} \in\right.$ $\{-1,0,1\}\}$, where the number of neighbors including itself is 27 .

As was shown in section 2, the number of cross-cell atom pairs is $q R_{c}^{3} \times 26 q R_{c}^{3}$ per cell, and it is impossible to store all the data in each core's private memory. Since the threebody interaction calculation also involves atoms in one cell and its 26 neighbor cells, it can also benefit from the cell-centered addressing for contiguous accessing of atomic data in a cell. Similarly, we group the neighbor atoms as well. Figure 4(b)depicts the data layout of neighbor atoms located in L2 cache or off-chip memory, where we group all the neighbor atomic data for the $i$-th cell together as L2_data_unit[ $i]$, which can be transferred to the SPM through a DMA like operation that utilizes high bandwidth provided in Godson- $T$.

\subsection{On-Chip Locality Aware Parallel Algorithm}

In this subsection, we present our on-chip locality aware parallel algorithm to enhance data reuse and further to alleviate the long latency to access the shared neighbor atomic data in L 2 cache or off-chip memory. The two-body force calculation involves core-core communication, and it may cause on-chip network congestion. Moreover, the access to L2 cache also goes through the on-chip network, which may introduce more congestion. Here, we propose a solution to enhance the data reuse and to reduce the remote shareddata memory accessing, thereby alleviating the long latency. Suppose that the atoms in a cell at core $i$ interacts with those in another cell at core $j$. In order to achieve on-chip locality for core $i$, we maximize the data reuse of cells from core $j$, and vice versa for core $j$. Our solution is first to construct a set of cell pairs $P C[c j]=\{c i 0, c i 1, ., c i k\}$, where $c j$ is the global index of the cell interacting with cells $c i 0, \ldots, c i k$ in core $i$. For example, suppose that core $i$ has cells $\{1,4,8\}$, and core $j$ has cells $\{2,5\}$. Also assume that cell 2 interacts with $\{1,4\}$ and that cell 5 interacts with $\{4,8\}$. Then we construct two cell pairs $P C[2]=\{1,4\}$ and $P C[5]=\{4,8\}$. We then use the corecore communication to transfer the atomic data according to the cell pairs from core $j$ to core $i$. If more than one cell are assigned to a core, then some neighbor cells are located in the same core, and thus no core-core communication is required.

The algorithm uses a preprocessing to collect the set of cell pairs. Since each cell only interacts with its 26 surrounding neighbors, the size of set $P C$ is expected to be less than $O\left(\frac{L}{P} \times 26\right)$. Since the calculation of neighbor's indices is done in $O(1)$ time using cell-centered addressing, the preprocessing requires $O\left(\frac{L}{P}\right)$ time and $O\left(\frac{L}{P}\right)$ space, which is negligible compared with the two-body interaction time $O\left(\frac{L}{P} \times q R_{c}^{3} \times 26 q R_{c}^{3}\right)$.

\section{Evaluation}

In this section, we present the experimental results and detailed analysis of the proposed MD optimization on a Godson-T many-core simulator.

Godson- $T$ is an on-going research project for building a petaflops supercomputer, and a real chip is expected to be shipped in late 2010. In order to evaluate its 
performance at early stage of the architectural development, we here employ an instructionlevel simulator. The Godson-T simulator is event-driven, cycle-accurate, executing both kernel and application codes, and has modeled all architectural features introduced previously. Since it is an instruction-level simulator, it can produce detailed traces and instruction mix of all executed instructions for any given application after execution. The toolchain on Godson- $T$ consists of a gcc-3.3 compiler and a thread execution runtime system, which provides a POSIX thread-like API. Moreover, since our ultimate objective is to build a large-scale parallel computer, where on-chip parallelism is of critical importance, we mainly focus on on-chip parallelization with a fixed problem size, and the speedup on $p$ cores is calculated by $S(p)=\frac{\text { Time }_{\text {one }} \text { core }}{}$ Time $e_{p_{-} \text {cores }}$, where Time $p_{p_{\text {ccores }}}$ rep-

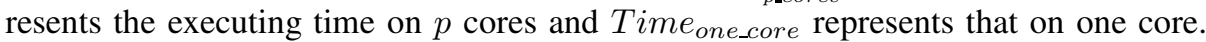
And strong-scaling parallel efficiency $E(p)$ is then defined as $E(p)=\frac{S(p)}{p}$ for a fixed problem size. In the following experiments, the MD simulation tested is for a silica system [5]. Within the DC framework, the whole system is divided into subsystems each containing 24,000 atoms as the fixed problem size for strong scalability analysis.

The experiments compare three incrementally improved versions of MD algorithm:

- optimization-1: implementation of ADC algorithm,

- optimization-2: implementation of ADC algorithm and data layout optimization,

- optimization-3: implementation of ADC algorithm, data layout optimization and on-chip locality-aware algorithm.

We have tested the scalability of the parallel algorithms. Figure 5 shows that optimization3 makes MD scale excellently with an on-chip strong-scaling parallel efficiency 0.92 on 64 cores while optimization-1 begins to deteriorate when the number of cores exceeds 32. It tells that optimizations which take advantage of architectural features to maximize data locality and exploit data reuse benefit scalability most, which is also evidenced by the running time in Fig. 6as optimization-3reduces the execution time by a factor of 2 .

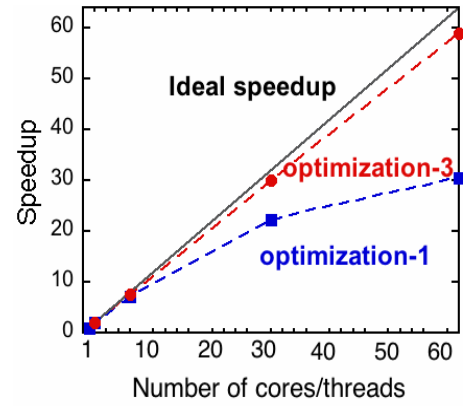

Fig. 5. Speedup as a function of the number of cores for optimization-1 and optimization-1

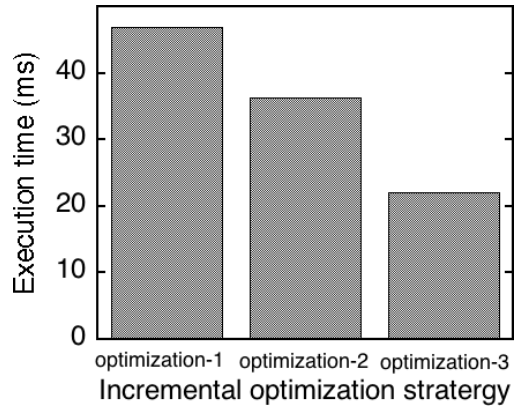

Fig. 6. Execution time in milliseconds on 64core Godson- $T$. Here, number $i$ represents optimization- $i(i=1,2,3,4)$

\section{Conclusion}

The emergence of many-core architecture has provided unprecedented computing power to computational scientists, and it is of great significance to exploit the computational 
power of such new platforms to improve the performance and scalability of large-scale scientific applications. In this paper, we have described our investigation of accelerating MD simulation on representative many-core architecture Godson- $T$. We have proposed a divide-and-conquer algorithm adaptive to the memory hierarchy to facilitate the onchip local memory, a novel data layout to improve data locality to alleviate the irregular memory accessing, an on-chip locality-aware parallel algorithm to enhance data reuse to amortize the long latency to access shared data. These techniques have made the parallel MD algorithm scale nearly linearly with the number of cores. Also we have found that the data locality and data reuse schemes taking advantage of explicit memory architecture and high-bandwidth on-chip network are essential to achieve high scalability. The contribution of this work lies not only in giving application scientists advice on how to optimize their applications utilizing architectural mechanisms, but also in guiding future hardware developments.

\section{References}

1. Fan, D.R., Yuan, N., Zhang, J.C., Zhou, Y.B., Lin, W., Song, F.L., Ye, X.C., Huang, H., Yu, L., Long, G.P., Zhang, H., Liu, L.: Godson-t: An efficient many-core architecture for parallel program executions. Journal of Computer Science and Technology 24(6), 1061-1073 (2009)

2. Grama, A., Karypis, G., Kumar, V., Gupta, A.: Introdution to Parallel Computing. Benjamin Cummings (2003)

3. Lin, W., Fan, D., Huang, H., Yuan, N., Ye, X.: A low-complexity synchronization based cache coherence solution for many cores. In: CIT 2009: Proceedings of the 2009 Ninth IEEE International Conference on Computer and Information Technology, pp. 69-75. IEEE Computer Society, Washington, DC, USA (2009)

4. Nakano, A., Kalia, R.K., Vashishta, P., Campbell, T.J., Ogata, S., Shimojo, F., Saini, S.: Scalable atomistic simulation algorithms for materials research. In: Supercomputing 2001: Proceedings of the 2001 ACM/IEEE Conference on Supercomputing (CDROM), p. 1. ACM, New York (2001)

5. Nomura, K., Seymour, R., Weiqiang, W., Dursun, H., Kalia, R.K., Nakano, A., Vashishta, P., Shimojo, F., Yang, L.H.: A metascalable computing framework for large spatiotemporal-scale atomistic simulations. In: IPDPS 2009: Proceedings of the IEEE International Symposium on Parallel \& Distributed Processing, pp. 1-10. IEEE Computer Society, Washington, DC, USA (2009)

6. Peng, L., Kunaseth, M., Dursun, H., Nomura, K., Wang, W., Kalia, R.K., Nakano, A., Vashishta, P.: A scalable hierarchical parallelization framework for molecular dynamics simulation on multicore clusters. In: Proceeding of International Conference on Parallel and Distributed Processing Techniques and Applications, pp. 97-103 (2009)

7. Wang, X., Gan, G., Manzano, J., Fan, D., Guo, S.: A quantitative study of the on-chip network and memory hierarchy design for many-core processor. In: ICPADS 2008: Proceedings of the 2008 14th IEEE International Conference on Parallel and Distributed Systems, pp. 689-696. IEEE Computer Society, Washington, DC, USA (2008)

8. Yu, L., Liu, Z., Fan, D., Song, F., Zhang, J., Yuan, N.: Study on fine-grained synchronization in many-core architecture. In: SNPD 2009: Proceedings of the 10th ACIS International Conference on Software Engineering, Artificial Intelligences, Networking and Parallel/Distributed Computing, pp. 524-529. IEEE Computer Society, Washington, DC, USA (2009) 